\title{
Orientation Histograms for Face Recognition
}

\author{
Friedhelm Schwenker, Andreas Sachs, Günther Palm, and Hans A. Kestler \\ University of Ulm \\ Department of Neural Information Processing \\ D-89069 Ulm \\ firstname.lastname@uni-ulm.de
}

\begin{abstract}
In this paper we present a method to recognize human faces based on histograms of local orientation. Orientation histograms were used as input feature vectors for a k-nearest neigbour classifier. We present a method to calculate orientation histograms of $n \times n$ subimages partitioning the $2 \mathrm{D}$-camera image with the segmented face. Numerical experiments have been made utilizing the Olivetti Research Laboratory (ORL) database containing 400 images of 40 subjects. Remarkable recognition rates of $98 \%$ to $99 \%$ were achieved with this extremely simple approach.
\end{abstract}

Keywords: Orientation histograms, Face recognition.

\section{Introduction}

Recognizing people in daily life is typically an effortless and unconscious task. The ease with which humans process such visual data leads to an underestimation of the complexity of this data processing. The identification of a human face poses several tests for any visual classifier system, for instance the high degree of similarity between faces from different persons, the influence to which lighting conditions can alter the 2D camara image of the face, or the large number of different views from which a face can be seen. In addition, there are many other influences on the facial appearance which may change from day to day, such as aging or makeup. For a robust real-life face recognition system all these problems have to be taken into account. In general a real world face recognition task requires the combination of at least three different pattern recognition tasks:

1. Tracking individuals moving around in the room

2. Detection and localization their faces

3. Recognition of the segmented faces

We concentrate on the third problem-recognizing faces of individuals. Over the last ten years, face recognition has become a special application area of computer vision, see for instance [13] for a survey. Sophisticated commercial systems perform face detection, image registration, and image recognition in real time. Many methods have been proposed for face recognition [13. Basically they can be divided into template matching based systems and geometrical local 
feature based systems. Typically, these systems perform the recognition task in two steps. First extracting characteristic features utilizing adaptive filters from the segmented camera image, and second classifying the feature vectors through a trainable classifier 6, 11 .

In this paper we propose a method to recognize human faces based on histograms of local orientation. These, so-called orientation histograms are then used as input feature vectors for a nearest neigbour classifier. Orientation histograms of images are very simple and fast to compute, so that real-time versions of this technique may be implemented. We present a method to calculate orientation histograms of $n \times n$ subimages of the camera image. Histogram techniques, particularly orientation histogramms, have successfully been applied to different visual 3D object recognition problems such as gesture recognition [12,10,7], and optical character recognition [9, 2].

Numerical experiments are made with the Olivetti Research Laboratory (ORL) database containing 400 images ofsubjects.

The paper is organized as follows: In Section 2 the ORL database is described. The orientation histogram technique is introduced in Section 3, the numerical experiments are presented in Section 4. Conclusions are given in Section 5.

\section{Dataset}

For the numerical evaluation the ORL database from the Olivetti Research Laboratory has been used. This database is available free of charge (see URL http:// www.cl.cam.ac.uk/Research/DTG/attarchive/facedatabase.html). Images of 40 distinct subjects with 10 recordings per person were taken. For a subsample of this database (one image per person) see Figure 1 .
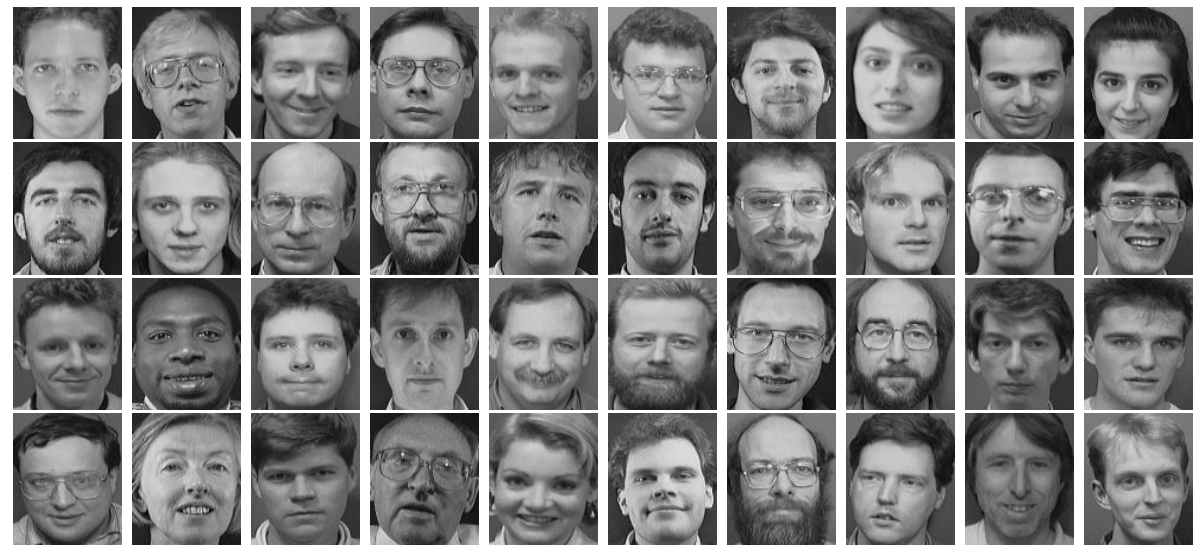

Fig. 1. A subsample of 40 images from the Olivetti Research Laboratory database. For each subject a single image is shown. The whole database consists of 10 images for each person. The images are gray scale images ( 8 bit) with a resolution of $92 \times 112$ pixels. 

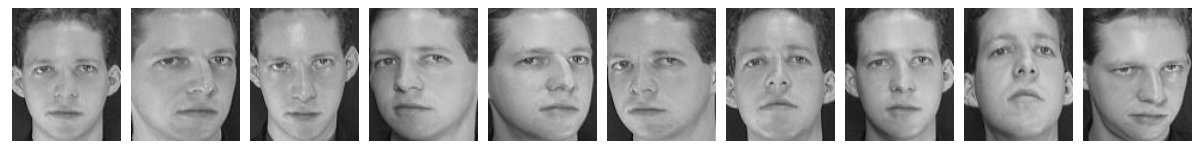

Fig. 2. Ten camera images taken from the same person showing the image variation within a class

The images show a variety of variations in facial expression (open/closed eyes) and facial details (glasses/no glasses). All these images were taken in front of a dark homogeneous background with the subjects in an up-right, frontal position. Images are grayscale images (8-bit) with a resolution of $92 \times 112$ pixel (see, [8 for further details on this database). To show the variation a subsample of 10 images taken from the same individual is given in Figure 2.

\section{Calculation of Orientation Histograms}

We now consider the problem of extracting orientation histogram features from camera images. Orientation histograms were introduced by Roth and Freeman to the literature of visual based pattern recognition by their use for hand gesture recognition [12. In this paper we propose to calculate orientation histograms on subimages. In the first step of this approach the whole image is divided into $n \times n$ sub-images, in Figure 3 the situation of a $2 \times 2$ partioning with non-overlapping sub-images is illustrated.

For each of these $n^{2}$ sub-image the orientation histogram of $m$ different directions (range: $0-2 \pi$, dark/light edges) is calculated 12 from the gray valued image. The orientation histograms of all sub-images are concatenated into the

A

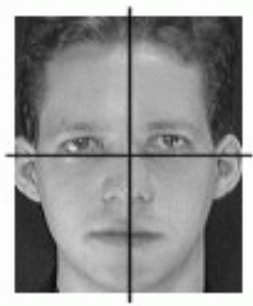

B

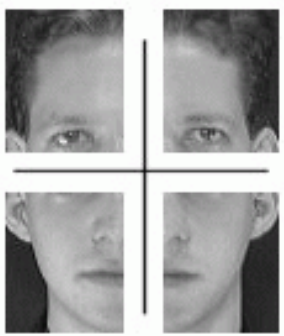

C

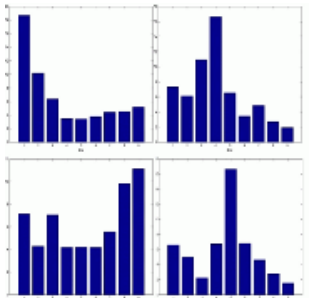

Fig. 3. Feature extraction process: The given camera image (A) is divided into overlapping or non-overlaping subimages (B). Then the orientation histograms within each of the $n^{2}$ subimages are calculated (C). The orientation of dark/light edges are quantized into a predefined number of $m$ bins. The feature extraction procedure leads to feature vectors consisting of $m n^{2}$ entries. These feature vectors serve as inputs into the classifier. 


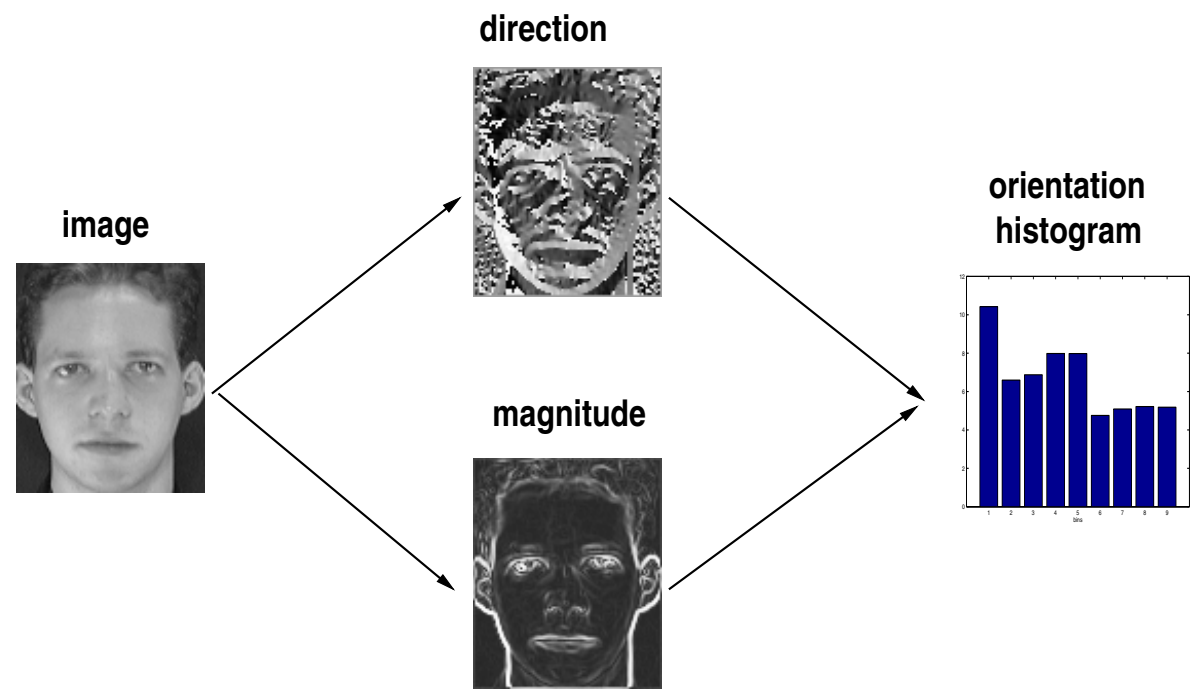

Fig. 4. Calculation of the orientation histogram (here with $m=10$ bins) of a gray scaled camera image. Local orientations are calculated and cummulated into the histogram. Entries in the histogram are determined through counting the number of angles falling into the respective bin.

characterizing feature vector which is the input to a classifier, e.g. a k-nearestneighbour classifier, artificial neural network based classifier, or decision tree.

For the calculation of the orientation histogram the gradient of an image $f(x, y)$ at location $(x, y)$ is given through the two dimensional vector

$$
\left(\begin{array}{l}
G_{x} \\
G_{y}
\end{array}\right)=\left(\begin{array}{l}
\frac{\partial f}{\partial x} \\
\frac{\partial f}{\partial y}
\end{array}\right) \approx\left(\begin{array}{l}
f * S_{x} \\
f * S_{y}
\end{array}\right)
$$

(here $*$ denotes the convolution operation). Gradient directions $\left(S_{x}, S_{y}\right)$ were then calculated through $3 \times 3$ Sobel operators. The gradient directions are calculated with respect to the $x$-axis:

$$
\alpha(x, y)=\operatorname{atan} 2\left(f * S_{y}, f * S_{x}\right)
$$

The atan2 function corresponds to the atan but additionally uses the sign of the arguments to determine the quadrant of the result 4, 5. The $m$ bins of the histogram all have equal size $2 \pi / m$. The histogram values are calculated by counting the number of angles falling into the respective bin. Histograms are normalized to the size of their sub-images. Figure 4 gives an overview.

\section{Numerical Experiments}

Our goal in this paper is not to develop elaborated machine learning techniques but to evaluate a feature extraction method for face recognition. Therefore, the 
nearest neighbour classifier has been used in our numerical evaluation which is the most elegant and most simple classifier [1].

Because we have to deal with a rather limited data set we utilized the crossvalidation method [3] to evaluate the proposed feature extraction methods. In the $k$-fold cross-validation testing procedure the data set is divided into $k$ disjoint subsets without considering image class. Then the 1-NN-classifier is determined $k$ times, each time using a version of the data set omitting exactly one of the $k$ subsets. The omitted subset is then used to test the 1-NN-classifier. Finally the achieved classification results are averaged over all $r$ classifier tests (generating the partitions randomly for each test). In Tables 1, 2, 3, and 4] the

Table 1. Recognition rates for the orientation histogram method calculated on the whole camera image, i.e. $n=1$. Results are shown for orientation quantization of $m=4,8,16,32,64$ bins per circle $[0,2 \pi)$. Means and standard deviation are given for 20 runs of ten-fold cross-validation experiments for the ORL database.

\begin{tabular}{|r|r|r|r|r|}
\hline$m=4$ & $m=8$ & $m=16$ & $m=32$ & $m=64$ \\
\hline $0.479(0.012)$ & $0.847(0.010)$ & $0.929(0.005)$ & $0.950(0.005)$ & $0.940(0.006)$ \\
\hline
\end{tabular}

Table 2. Classification accuracies of the orientation histogram method calculated on the ORL database. Means and standard deviation are given for 20 runs of ten-fold crossvalidation experiments.

\begin{tabular}{|r|r|r|r|}
\hline & $m=8$ & $m=16$ & $m=32$ \\
\hline $2 \times 2$ & $0.979(0.004)$ & $0.985(0.004)$ & $0.982(0.005)$ \\
$3 \times 3$ & $0.976(0.004)$ & $0.984(0.003)$ & $0.983(0.002)$ \\
\hline
\end{tabular}

Table 3. Recognition rates for the orientation histogram method with $2 \times 2$ and $3 \times 3$ subimages. The overlap between neighbouring subimages was $o=0.25$. Means and standard deviations calculated for 20 runs of a 10 -fold crossvalidation experiment on the ORL database.

\begin{tabular}{|r|r|r|r|}
\hline & $m=8$ & $m=16$ & $m=32$ \\
\hline $2 \times 2$ & $0.971(0.005)$ & $0.974(0.006)$ & $0.976(0.006)$ \\
$3 \times 3$ & $0.980(0.005)$ & $0.985(0.003)$ & $0.987(0.002)$ \\
\hline
\end{tabular}

Table 4. Recognition rates for the orientation histogram method with $2 \times 2$ and $3 \times 3$ subimages. The overlap between neighbouring subimages was $o=0.5$. Means and standard deviations calculated for 20 runs of a 10 -fold crossvalidation experiment on the ORL database.

\begin{tabular}{|r|r|r|r|}
\hline & $m=8$ & $m=16$ & $m=32$ \\
\hline $2 \times 2$ & $0.964(0.007)$ & $0.968(0.004)$ & $0.974(0.006)$ \\
$3 \times 3$ & $0.979(0.004)$ & $0.990(0.004)$ & $0.983(0.006)$ \\
\hline
\end{tabular}


calculated means of the $r=20$ ten-fold cross-validation testings on the ORL database are given.

In the first series of experiments the orientation hiostograms have been determinted from the whole camera image for a quantization of $m=4,8,16,32,64$ bins, see Figure 4 At the first sight, the recognition rate increases with increasing number of orientation bins. We found a maximal recognition rate of $95 \%$ for a resolution of $m=32$ bins.

The second series of experiments is to investigate the influence of the subimage partitioning. Images were divided into $n \times n$ non-overlaping subimages, again with different quantization levels for orientation. Results of our numerical experiments for the sub-image approach dividing the image into $n \times n$ sub-images with $n=2,3$ and with o resolution in the range for $m=8$ to $m=32$ histogram bins are given in Table2 The accuracy for each parameter combination is $>97 \%$ this significantly higher as for the basic approach. In the next step overlapping sub-images were considered and results for two different degrees of overlap, $o=$ 0.25 and $o=0.5$, are presented in Table 3 and $0, o=\alpha$ stands for the overlap of neigbouring sub-images. We found approximately the same recognition rates as for non-overlapping sub-images, but at least for some parameter combinations the performance was slightly improved, and we achieved the best result (error rate $1 \%$ ) for $3 \times 3$ sub-images with a resolution of $m=16$ orientation histogram bins and an overlap degree of $o=1 / 2$.

\section{Conclusion}

In this paper we have applied the very simple method of orientation histograms to the problem of face recognition. For camera images of human faces the histograms of local orientations were calculated on $n \times n$ subimages. These extracted feature vectors were then concatenated and used as the input vectors of nearest neighbour classifiers. Remarkable recognition rates of $98 \%$ to $99 \%$ were achieved with this extremely simple approach and compare very well to the $3.8 \%$ error rate reported by Lawrence et.al. [8] on the same data. They used more complex classifiers based on convolution neural networks with features derived from the whole image rather then the sub-image approach we applied. This lets us conclude that image pre-processing and feature extraction are extremely important in face recognition and that our method of orientation histograms of sub-images seems feasible in this setting.

\section{References}

1. Richard O. Duda, Peter E. Hart, and David G. Stork. Pattern Classification. John Wiley \& Sons, New York, 2001.

2. H. Fujisawa and C.-L. Liu. Directional pattern matching for character recognition revisited. In Proc. of the 7th ICDAR, pages 794-798, 2003.

3. K. Fukunaga. Introduction to Statistical Pattern Recognition. Academic Press, 1991. 
4. P. Haberäcker. Practical Handbook of Image Processing for Scientific Applications. Hanser-Verlag, 1995.

5. B. Jähne. Practical Handbook of Image Processing for Scientific Applications. CRC Press, 1997.

6. K. Jonsson, J. Kittler, Y. Li, and J. Matas. Support vector machines for face authentication. In T. Pridmore and D. Elliman, editors, Proceedings of the BMVC'99, pages 543- 553, 1999.

7. H.A. Kestler, S. Simon, A. Baune, F. Schwenker, and G. Palm. Object Classification Using Simple, Colour Based Visual Attention and a Hierarchical Neural Network for Neuro-Symbolic Integration. In W. Burgard, T. Christaller, and A.B. Cremers, editors, KI-99: Advances in Artificial Intelligence, pages 267-279. Springer Verlag, 1999.

8. Steve Lawrence, C. Lee Giles, A. C. Tsoi, and A. D. Back. Face recognition: A convolutional neural network approach. IEEE Transactions on Neural Networks, 8(1):98-113, 1997.

9. C.-L. Liu, K. Nakashima, H. Sako, and H. Fujisawa. Handwritten digit recognition: benchmarking of state-of-the-art techniques. Pattern Recognition, 36(10):2271$2285,2003$.

10. B. Mel. Seemore: Combining colour, shape, and texture histogramming in a neurally-inspired approach to visual object recognition. Neural Computation, 9:777-804, 1997.

11. C. Nakajima, M. Pontil, B. Heisele, and T. Poggio. People recognition in image sequences by supervised learning. Technical report 1633/CBCL Memo No.133, MIT AI, 2000.

12. M. Roth and W.T. Freeman. Orientation histograms for hand gesture recognition. Technical Report 94-03, Mitsubishi Electric Research Laboratorys, Cambridge Research Center, 1995.

13. W. Zhao, R. Chellappa, P.J. Phillips, and A. Rosenfeld. Face recognition: a literature survey. ACM Computing Surveys, 35(4):399-458, 2003. 